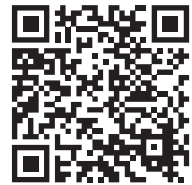

Enero - Marzo 2022 Vol. 2, núm. 1 / pp. 6-11

\title{
Carcinoma epidermoide de cavidad oral en edad pediátrica. A propósito de un caso. Revisión de la literatura
}

\author{
Epidermoid carcinoma of the oral cavity in pediatric age. \\ Apropos of a case. Review of the literature \\ Julio Acero, ${ }^{\star}$ Álvaro Pastor, ${ }^{\ddagger}$ Alberto Haddad, ${ }^{\S}$ \\ Elena Baranda, ${ }^{\S}$ Ángela Bueno, ${ }^{\S}$ Manuel Picón,, Fernando Almeida ${ }^{\S}$
}

Palabras clave:

Cáncer oral,

edad infantil,

reconstrucción

mandibular.

Keywords:

Oral Cancer,

childhood, mandibular

reconstruction.

\footnotetext{
* Jefe de Servicio de Cirugía Maxilofacial. Profesor Titular de Cirugía. Hospital Universitario Ramón y Cajal. Hospital Universitario Puerta de Hierro. Universidad de Alcalá. Instituto Ramón y Cajal de Investigación (IRYCIS), Madrid. España. ‡Especialista Cirugía Oral y Maxilofacial, Hospital Universitario Quirón, Madrid, España. Especialista Cirugía Oral y Maxilofacial, Hospital Universitario Ramón y Cajal. Hospital Universitario Puerta mandibulectomía segmentaria izquierda con márgenes oncológicos de seguridad, disección cervical funcional anterolateral izquierda y reconstrucción con colgajo microvascularizado de peroné fijado con barra de titanio. Se utilizaron guías de corte y planificación virtual. A los 24 meses de seguimiento postoperatorio, no presenta recidiva locorregional ni a distancia. Conclusiones: El carcinoma epidermoide de cavidad oral en edad pediátrica tiene una baja incidencia, precisando un manejo y reconstrucción óptimos. El colgajo de peroné podría ser la opción más indicada para la reconstrucción mandibular en edad infantil. La preservación del cóndilo mandibular podría favorecer el crecimiento mandibular del paciente durante el desarrollo.
} de Hierro. Madrid, España.

Recibido: 01/11/2021 Aceptado: 10/11/2021

doi: $10.35366 / 103403$ dx.doi.org/10.35366/103403

\section{RESUMEN}

Introducción: El carcinoma epidermoide de cavidad oral es una entidad frecuente en la patología maligna de cabeza y cuello del adulto; sin embargo, es extremadamente raro en edad pediátrica. El tratamiento y la reconstrucción de los pacientes en edad infantil suponen un reto por su alta complejidad y los pocos casos descritos hasta la fecha en la literatura. Material y métodos: Se presenta un caso clínico de carcinoma epidermoide mandibular en paciente de edad pediátrica. Se realiza diagnóstico mediante estudio histopatológico y pruebas radiológicas, y se lleva a cabo una planificación virtual para la reconstrucción mandibular. Se realiza revisión de la literatura al tratarse de una entidad poco frecuente. Resultados: Varón de nueve años de edad de origen asiático que acudió a nuestras consultas por clínica de supuración en región mandibular izquierda próxima a primer molar definitivo, de aproximadamente dos meses de evolución. Se realizó toma de biopsia con resultado anatomopatológico de carcinoma epidermoide. Se presentó el caso en el Comité de Tumores de Cabeza y Cuello, decidiéndose tratamiento quirúrgico:

\section{ABSTRACT}

Introduction: Oral cavity squamous cell carcinoma is a frequent entity in adult malignant head and neck pathology; however, it is extremely rare in pediatric age. The treatment and reconstruction of pediatric patients is a challenge due to its high complexity and the few cases described to date in the literature. Material and methods: A clinical case of mandibular squamous cell carcinoma in a pediatric patient is presented. Diagnosis is made by histopathological study and radiological tests, and a virtual planning for mandibular reconstruction is performed. A review of the literature is performed since it is a rare entity. Results: A 9-yearold male of Asian origin came to our office for clinical symptoms of suppuration in the left mandibular region close to the first definitive molar, of approximately 2 months of evolution. A biopsy was performed with an anatomopathological result of squamous cell carcinoma. The case was presented to the Head and Neck Tumors Committee and surgical treatment was decided: left segmental mandibulectomy with oncologic safety margins, left anterolateral functional cervical dissection and reconstruction with microvascularized fibula flap fixed with titanium rod. Cutting guides and virtual planning were used. At 24 months postoperative followup there was no locoregional or distant recurrence. Conclusions: Oral cavity squamous cell carcinoma in pediatric age has a low incidence, requiring optimal management and reconstruction. The fibula flap could be the most indicated option for mandibular reconstruction in children. The preservation of the mandibular condyle could favor the mandibular growth of the patient during development.

Citar como: Acero J, Pastor Á, Haddad A, Baranda E, Bueno Á, Picón M, et al. Carcinoma epidermoide de cavidad oral en edad pediátrica. A propósito de un caso. Revisión de la literatura. Lat Am J Oral Maxillofac Surg. 2022; 2 (1): 6-11. https:// 


\section{INTRODUCCIÓN}

El carcinoma epidermoide (CE) de cavidad oral es una entidad rara en pacientes en edad pediátrica, siendo más frecuente encontrarla en adultos varones mayores de 60 años y habitualmente asociada al consumo de alcohol y tabaco. Éstos factores de riesgo no se hallan en edad infantil, por lo que en su origen etiopatogénico se ven involucrados otros factores, entre los que se encuentran los de tipo genético (anemia de Fanconi, síndrome de Bloom, disqueratosis congénita) o de tipo ambiental. En el grupo de pacientes menores de 40 años, el CE supone aproximadamente $4 \%$ de todos los cánceres de cavidad oral, proporción mucho menor si limitamos el período a la edad pediátrica, lo que la convierte en una entidad extremadamente rara. ${ }^{1}$ De acuerdo con la literatura, ${ }^{2}$ esta enfermedad es particularmente agresiva en jóvenes y se asocia con menores tasas de supervivencia en comparación con los adultos. Cuando esta entidad afecta a la mandíbula además de a las partes blandas orales, el mayor reto de la cirugía reconstructiva consiste en la restauración de la masticación, deglución y el habla, afectando lo mínimo posible a la estética facial. En niños, además de todo lo anterior, se añade la particularidad de que la mandíbula ha de desarrollarse sincrónicamente y de forma armónica con el maxilar y la base de cráneo. Otros factores a tener en cuenta en la edad pediátrica son la erupción correcta de la dentición definitiva y la morbilidad de la zona donante. Con respecto a las terapias adyuvantes (quimioterapia y radioterapia), parece demostrado que se presentan más complicaciones que en el adulto, ya que a largo plazo pueden producir un aumento de la incidencia de tumores secundarios y secuelas funcionales. ${ }^{3}$ Debido a la escasa incidencia de este tipo de tumores en niños, resulta complicado identificar las formas de presentación, asî como establecer los factores pronósticos y desarrollar las mejores modalidades de tratamiento, por lo que es imprescindible contar con un equipo multidisciplinar (cirujanos maxilofaciales, oncólogos médicos y radioterapeutas, radiólogos, anatomopatólogos, etcétera). En este contexto, el objetivo fundamental es

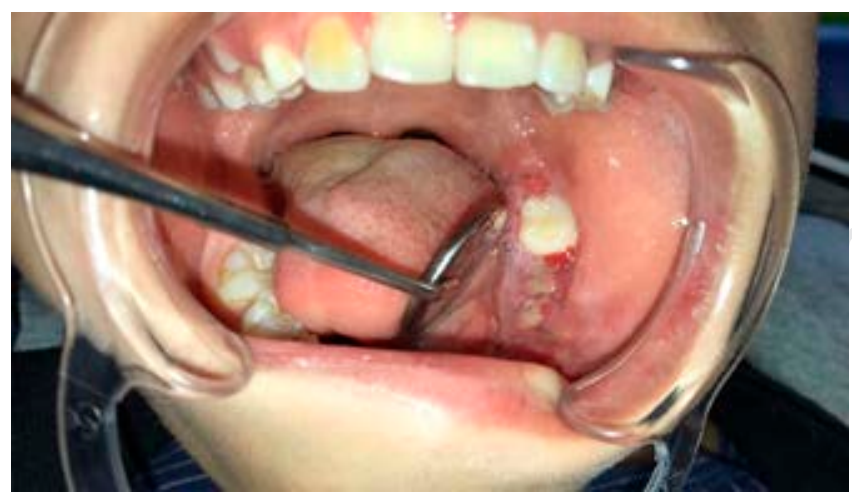

Figura 1: Imagen clínica del paciente. Aspecto intraoral.

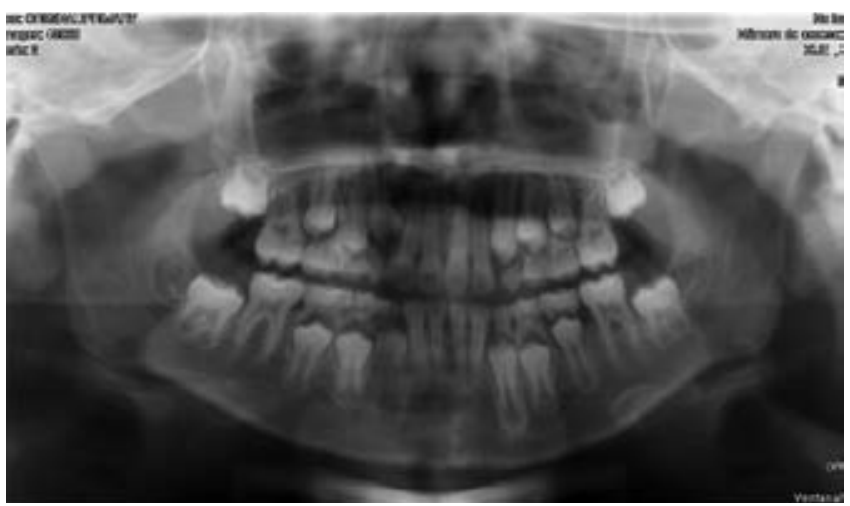

Figura 2: Ortopantomografía.

desarrollar una minuciosa planificación tanto del tratamiento oncológico como de la ulterior reconstrucción. ${ }^{4}$

\section{MATERIAl y mÉTOdos}

Presentamos el caso clínico de un varón de nueve años diagnosticado con carcinoma epidermoide de encía mandibular izquierda. Para el diagnóstico se realizaron: biopsia incisional, ortopantomografía, tomografía computada (TC) cervicofacial, resonancia magnética (RMN) facial y TC de miembros inferiores. Se realizó cirugía resectiva del tumor, previa planificación virtual e impresión 3D de guías de corte para la resección y reconstrucción mandibular inmediata con colgajo microquirúrgico osteo-cutáneo de peroné.

Se realizó revisión sistemática de la literatura hasta la fecha de publicación de este artículo (Pubmed), al tratarse de una entidad con muy baja incidencia.

\section{Resultados}

Niño de nueve años de origen asiático valorado en las consultas externas de Cirugía Maxilofacial del Hospital Universitario «Ramón y Cajal», donde acude por cuadro clínico de inflamación, dolor y supuración a nivel del reborde mandibular izquierdo de unos dos meses de evolución (Figura 1). El paciente había sido valorado previamente por su dentista, siendo tratado con antibioterapia al considerar una infección dental con origen en pieza 36 como primera opción diagnóstica. Aportaba una ortopantomografía previa sin hallazgos relevantes (Figura 2).

A su llegada a nuestro centro, presentaba inflamación paramandibular izquierda y supuración intraoral, junto con lesión ulcerada exofítica por las vertientes vestibular y lingual del tercer cuadrante en torno a pieza 36 . Se realizó una toma de biopsia incisional bajo anestesia local, que se envió para estudio anatomopatológico. El estudio de la muestra informó de «fragmento de encía con signos inflamatorios y un área de proliferación epitelial escamosa que plantea el diagnóstico con 
carcinoma epidermoide (CE) bien diferenciado con formación de quistes (carcinoma cuniculatum)». El servicio de Anatomía Patológica recomendó en ese momento la vigilancia estrecha de la lesión ante la baja probabilidad de tratarse de un CE dada la edad del paciente. Ante la ausencia de mejoría del cuadro clínico del paciente, y en vista del resultado anatomopatológico, se remitió a la sección de Oncología del servicio de Cirugía Maxilofacial, donde finalmente se realizó una biopsia amplia de la lesión y exodoncia de las piezas 35, 36 y 74

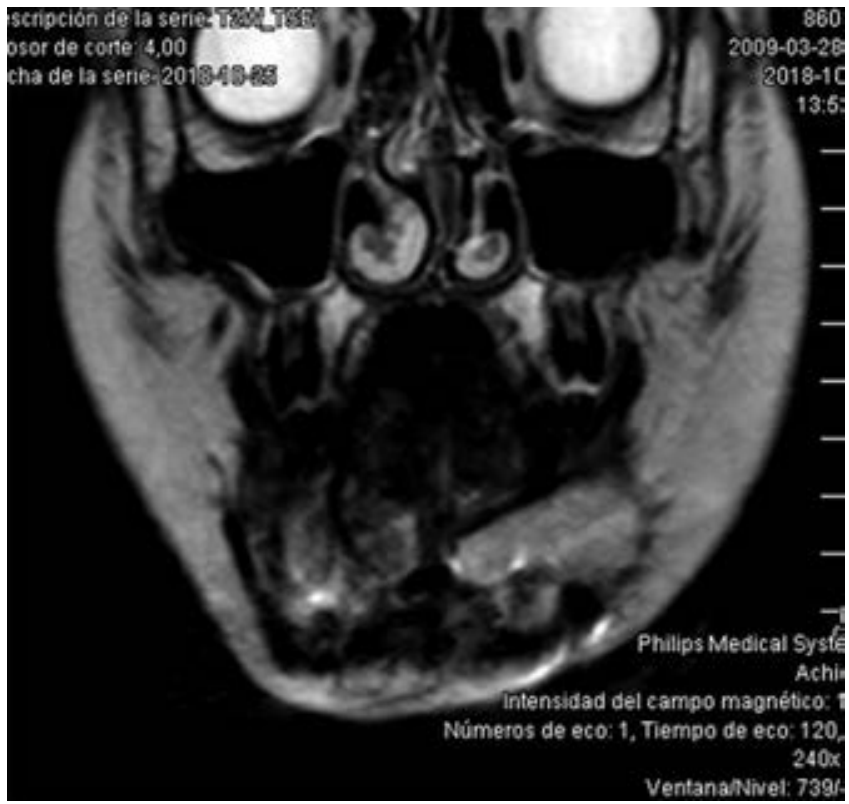

Figura 3: Resonancia magnética preoperatoria. bajo anestesia general. El resultado de esta segunda biopsia confirmó el diagnóstico de CE infiltrante bien diferenciado, por lo que se solicitaron las pruebas complementarias radiológicas para el estudio locorregional del tumor y descartar la afectación a distancia.

La RMN facial informaba de cambios postquirúrgicos en hemimandíbula izquierda con tejido de partes blandas en el lecho quirúrgico, hipointenso en secuencias $\mathrm{T} 1$ e hiperintenso en T2, con realce tras la administración de contraste, que podrían corresponder con cambios inflamatorios postquirúrgicos sin poder descartar persistencia de tejido tumoral. Se asociaba con una alteración de la señal en la médula ósea de la hemimandíbula izquierda. Desde el lecho quirúrgico, la lesión alcanzaba el proceso alveolar de la pieza 33 anteriormente, y de manera posterior se extendía hasta los procesos alveolares de las piezas 37 y 38 , sin superar esta última. Asimismo, englobaba el canal del nervio dentario inferior sin alcanzar la espina de Spix. En las secuencias postcontraste no se observaron signos de diseminación perineural (Figura 3). Se solicitó también un TC cervicofacial con hallazgos similares a los descritos en la RMN, no siendo identificados ganglios patológicos en el área cervical.

De ésta forma, el cuadro clínico fue estadiado como carcinoma epidermoide mandibular izquierdo en edad pediátrica (cT4NOMO) y se presentó en el Comité de Tumores de Cabeza y Cuello del Hospital «Ramón y Cajal», decidiéndose llevar a cabo tratamiento quirúrgico con intención curativa. Previamente a la intervención quirúrgica, la planificación del caso incluyó la cirugía virtual de la resección mandibular y la reconstrucción del caso, usando un software en 3D con las imágenes del cráneo y el peroné del paciente. El diseño de la reconstrucción de la neomandíbula se realizó incluyendo dos fragmentos de peroné (Figura 4). Tanto para llevar a cabo
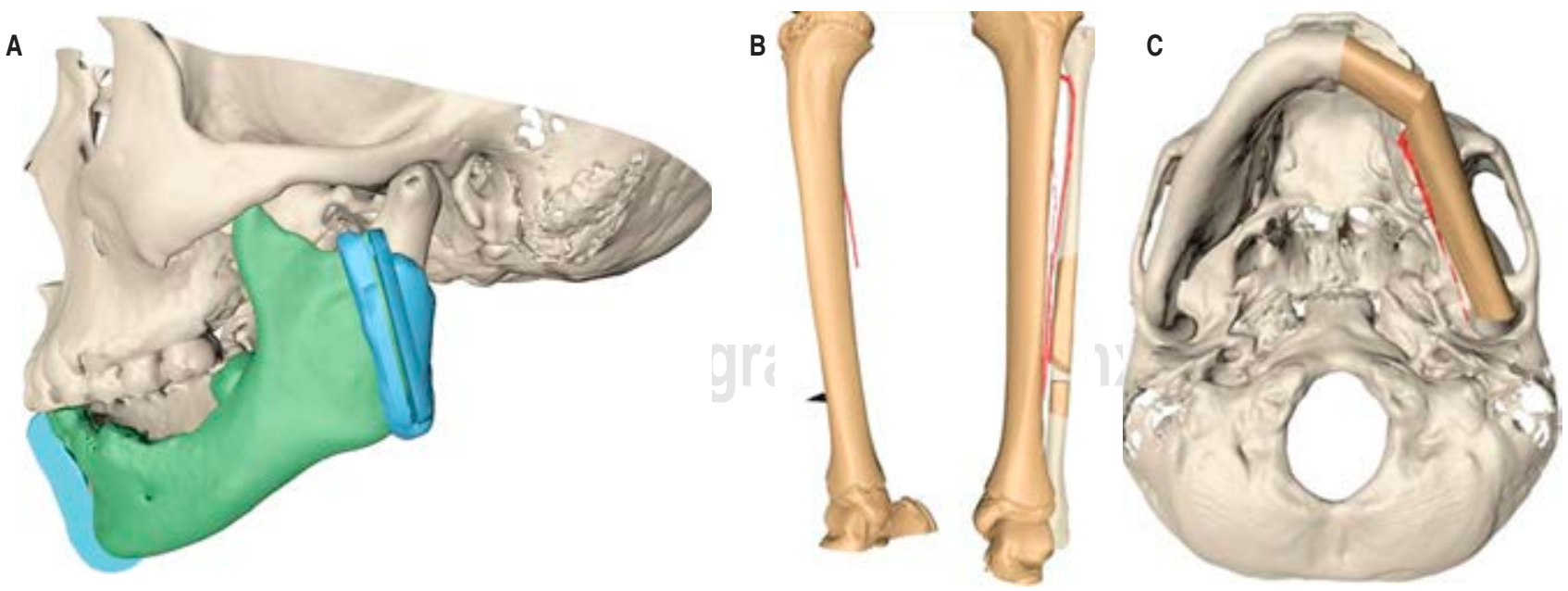

Figura 4: A) Planificación resección segmentaria mandibular. B,C) Planificación virtual para reconstrucción con colgajo microquirúrgico de peroné. 


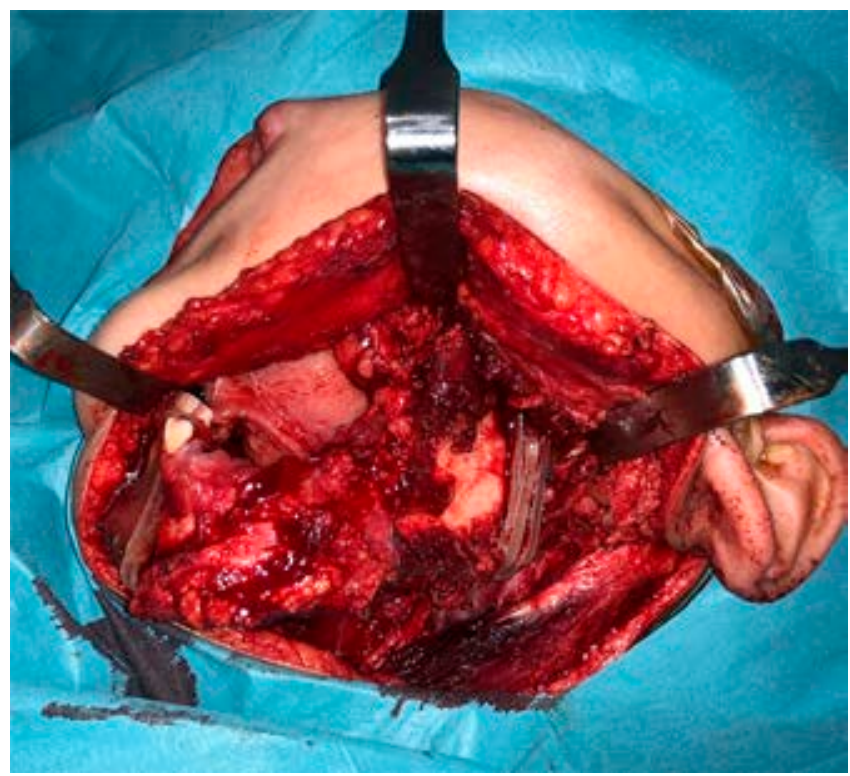

Figura 5: Imagen intraoperatoria: hemimandibulectomía izquierda con las guías de corte adaptadas a la mandíbula.

los cortes en la mandíbula como los cortes en el peroné, se fabricaron guías de corte específicas y para la fijación de los fragmentos de peroné previamente descritos se diseñó una barra de reconstrucción mandibular en titanio personalizada (custom-made). El tratamiento quirúrgico consistió en resección tumoral con márgenes de seguridad (mandibulectomía segmentaria izquierda) desde la pieza 31 hasta la práctica totalidad de la rama mandibular izquierda, respetando el cóndilo mandibular, disección cervical funcional anterolateral izquierda y reconstrucción con colgajo osteocutáneo microvascularizado de peroné izquierdo (Figuras 5 y 6). El postoperatorio cursó sin complicaciones.

El resultado anatomopatológico de la pieza quirúrgica definitiva informó de CE bien diferenciado con patrón histológico similar a carcinoma verrucoso/carcinoma cuniculatum (Figura 7), con márgenes libres de tumor a una distancia de 0.8-1 cm, así como un total de 32 ganglios cervicales con estudio negativo para malignidad. Con este resultado presentado nuevamente en el Comité de Tumores, se decidió no añadir tratamiento adyuvante y realizar vigilancia estrecha del paciente.

A los dos años de seguimiento postquirúrgico, el paciente no presenta recidiva locorregional ni a distancia (Figura 8). El paciente se ha reincorporado completamente a su vida escolar y social, se alimenta por vía oral con facilidad y no ha presentado morbilidad significativa en la zona donante. No existe tampoco una limitación del crecimiento ni para la apertura oral y oclusalmente se encuentra bien, con líneas medias maxilar y mandibular centradas. La rehabilitación dental así como posible retirada de placa de fijación serán planteadas más adelante cuando el paciente finalice su desarrollo completo.

\section{Discusión}

El CE es la estirpe histológica más común de los cánceres localizados en cabeza y cuello; sin embargo, el CE oral en edad pediátrica es poco común, representa aproximadamente $4 \%$ de los casos de cabeza y cuello. ${ }^{5,6}$ Dada la edad de este grupo

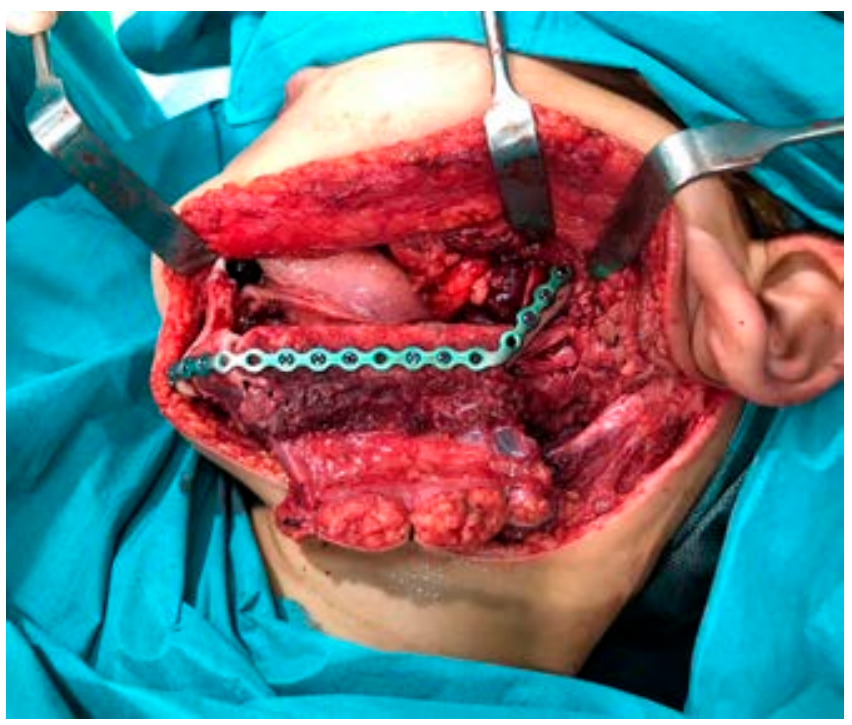

Figura 6: Reconstrucción mandibular con colgajo microvascularizado de peroné.

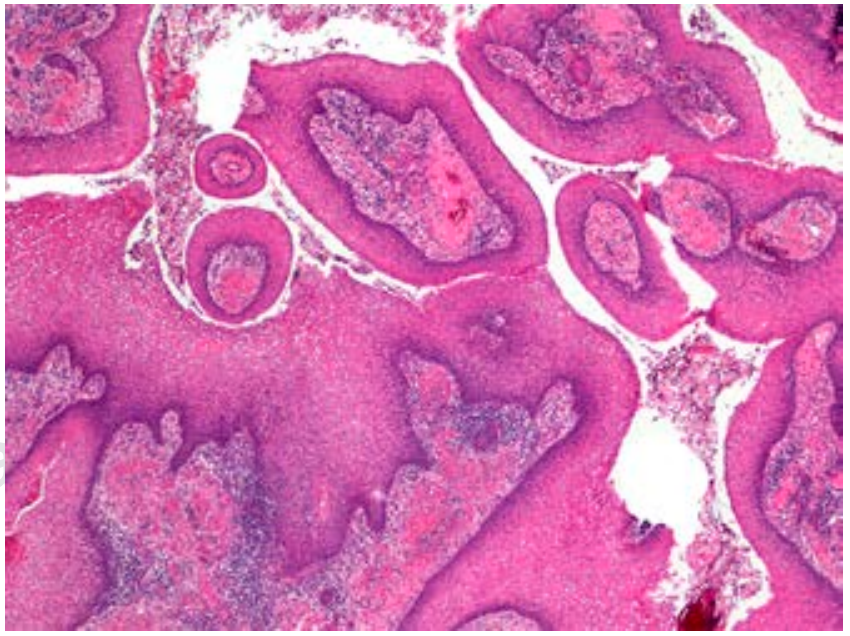

Figura 7: Imagen histopatológica. El tumor crece formando pseudopapilas y cavidades o túneles intercomunicados y rellenos de queratina y detritus celulares. 

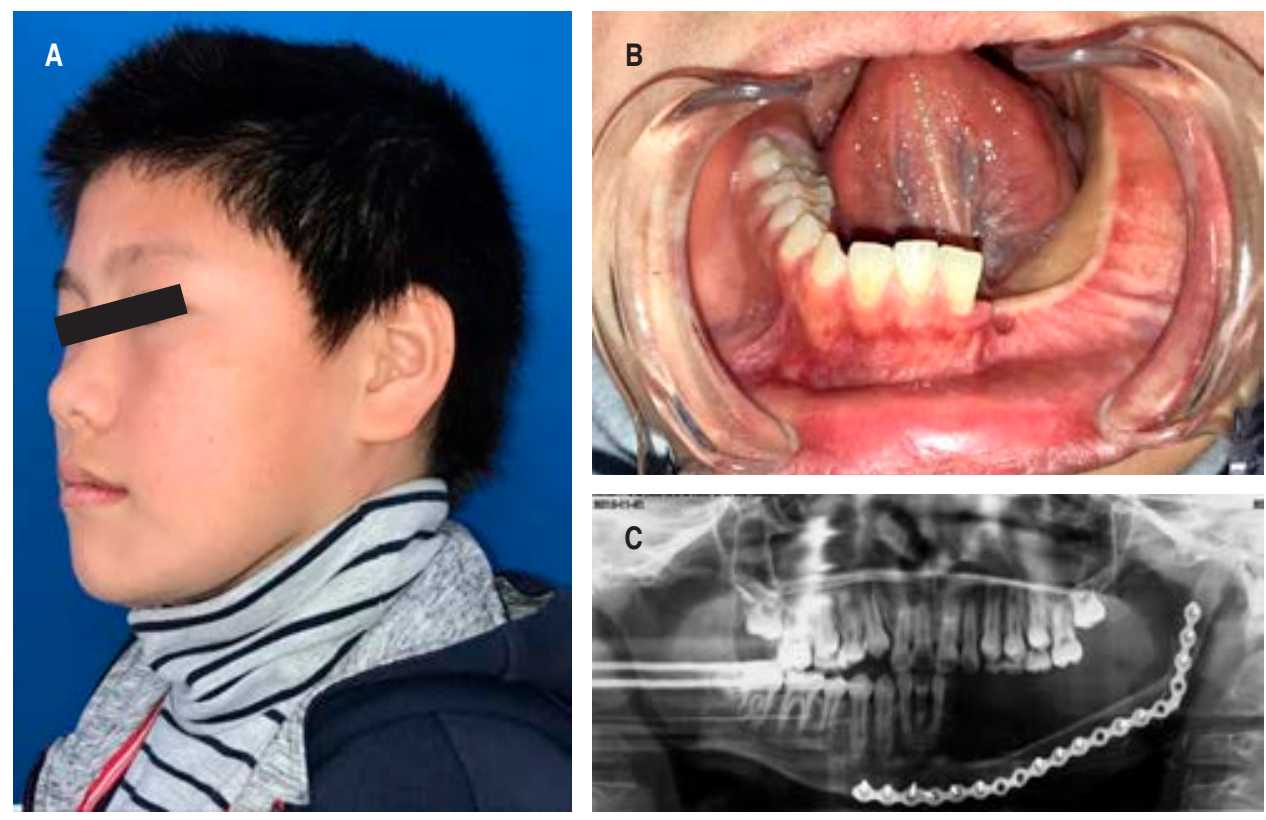

Figura 8:

A,B) Imagen clínica facial e intraoral. C) Ortopantomografía postquirúrgica.

de pacientes, es imprescindible un manejo y reconstrucción óptimos. Es de común acuerdo, según diversos estudios, que debido a la agresividad que presentan estos tumores en edad infantil, el diagnóstico en las fases más iniciales es primordial (aunque resulta difícil pensar en ellos como primera opción diagnóstica dada la baja incidencia), influyendo en el pronóstico y en el curso de la enfermedad.

A pesar de tratarse de una entidad maligna, nuestro paciente presentó signos similares a los que podría presentar una lesión benigna mandibular, como inflamación y tumoración de la zona. Sin embargo, otros signos o síntomas habituales como dolor persistente, movilidad dental o afectación del nervio dentario inferior no se manifestaron.

Los tumores malignos pediátricos más frecuentes como los sarcomas son habitualmente tratados con cirugía más conservadora y quimioterapia adyuvante, llegando a conseguir altas tasas de curación y periodos libres de enfermedad. ${ }^{7} \mathrm{Sin}$ embargo, el CE presenta otro tipo de comportamiento que hace necesaria una cirugía ablativa inicialmente, añadiendo tratamiento adyuvante en función de la anatomía patológica definitiva. ${ }^{8,9}$ En casos agresivos suele optarse por la quimioterapia para evitar las complicaciones locorregionales o a distancia de la radioterapia, tanto a corto como a largo plazo. ${ }^{6}$

La reconstrucción mandibular con tejido óseo en niños tras la cirugía resectiva resulta imprescindible, dando prioridad en resecciones segmentarias complejas a los colgajos microvascularizados como primera opción de tratamiento reconstructivo. ${ }^{10,11}$ En nuestra opinión, la reconstrucción mandibular en edad pediátrica mediante colgajo de cresta iliaca puede estar contraindicada debido a las posibles alteraciones en el crecimiento esquelético del paciente, por lo que el peroné parece ser la mejor opción en cuanto a aporte de hueso y menor morbilidad de la zona donante. ${ }^{12}$ Asimismo, la preservación del cóndilo mandibular en el paciente podría favorecer el correcto desarrollo mandibular y el crecimiento de forma armónica con el resto del esqueleto facial a lo largo del tiempo. La rehabilitación dental de estos casos con implantes osteointegrados no debe plantearse hasta haber finalizado completamente el período de crecimiento. ${ }^{13}$

\section{Conclusiones}

El carcinoma epidermoide de cavidad oral en edad pediátrica tiene una baja incidencia, precisando un manejo y reconstrucción óptimos. El colgajo de peroné podría ser la opción más indicada para la reconstrucción mandibular en edad infantil. La preservación del cóndilo mandibular podría favorecer el crecimiento mandibular del paciente durante el desarrollo, pero se requiere más información y estudios en la literatura para hacer de esta práctica un estándar viable a ser repetido en casos parecidos; sin embargo, con base en la evidencia presentada, el protocolo de trabajo expuesto representa una opción viable y estable en el tiempo.

\section{RefERENCIAS}

1. Bhanu PV, Mallick S, Upadhyay AD, Rath GK. Systematic review and individual patient data analysis of pediatric head and neck squamous cell carcinoma: An analysis of 217 cases. Int J Pediatr Otorhinolaryngol. 2017; 92: 75-81. doi: 10.1016/j.ijporl.2016.11.005. Epub 2016 Nov 12. PMID: 28012539.

2. Chow CW, Tabrizi SN, Tiedemann K, Waters KD. Squamous cell carcinomas in children and young adults: a new wave of a very rare 
tumor? J Pediatr Surg. 2007; 42 (12): 2035-2039. doi: 10.1016/j. jpedsurg.2007.08.025. PMID: 18082703.

3. Dombrowski ND, Wolter NE, Irace AL, Robson CD, Perez-Atayde AR, Mack JW, Rahbar R. Squamous cell carcinoma of the head and neck in children. Int J Pediatr Otorhinolaryngol. 2019; 117: 131-137. doi: 10.1016/j.ijporl.2018.11.019. Epub 2018 Nov 20. PMID: 30579067.

4. George AP, Markiewicz MR, Garzon S, Choi DK. Adolescent and Young Adult Oral Maxillofacial Tumors: A Single-Institution Case Series and Literature Review. J Adolesc Young Adult Oncol. 2020; 9 (2): 307-312. doi: 10.1089/jayao.2019.0111. Epub 2019 Dec 20. PMID: 31859579; PMCID: PMC7476397.

5. Smith A, Petersen D, Samant S, Ver Halen JP. Pediatric mandibular reconstruction following resection of oral squamous cell carcinoma: a case report. Am J Otolaryngol. 2014; 35 (6): 826-828. doi: 10.1016/j. amjoto.2014.07.004. Epub 2014 Jul 17. PMID: 25123780.

6. Bhanuprasad V, Mallick S, Bhasker S, Mohanti BK. Pediatric head and neck squamous cell carcinoma: Report of 12 cases and illustrated review of literature. Int J Pediatr Otorhinolaryngol. 2015; 79 (8): 1279-1282. doi: 10.1016/j.ijporl.2015.05.031. Epub 2015 May 30. PMID: 26072014.

7. Stolk-Liefferink SA, Dumans AG, van der Meij EH, Knegt PP, van der Wal KG. Oral squamous cell carcinoma in children; review of an unusual entity. Int J Pediatr Otorhinolaryngol. 2008; 72 (1): 127 131. doi: 10.1016/j.ijporl.2007.09.006. Epub 2007 Oct 29. PMID: 18029030.

8. Benoit MM, Vargas SO, Bhattacharyya N, McGill TA, Robson CD, Ferraro $\mathrm{N}$, et al. The presentation and management of mandibular tumors in the pediatric population. Laryngoscope. 2013; 123 (8):
2035-2042. doi: 10.1002/lary.24020. Epub 2013 May 13. PMID: 23670306.

9. Sidell D, Nabili V, Lai C, Cheung G, Kirsch C, Abemayor E. Pediatric squamous cell carcinoma: Case report and literature review. Laryngoscope. 2009; 119 (8): 1538-1541. doi: 10.1002/lary.20531. PMID: 19522006.

10. Modh A, Gayar OH, Elshaikh MA, Paulino AC, Siddiqui F. Pediatric head and neck squamous cell carcinoma: Patient demographics, treatment trends and outcomes. Int J Pediatr Otorhinolaryngol. 2018; 106: 21-25. doi: 10.1016/j.ijporl.2017.12.032. Epub 2018 Jan 3. PMID: 29447885.

11. Genden EM, Buchbinder D, Chaplin JM, Lueg E, Funk GF, Urken ML. Reconstruction of the pediatric maxilla and mandible. Arch Otolaryngol Head Neck Surg. 2000; 126 (3): 293-300. doi: 10.1001/ archotol.126.3.293. PMID: 10722000.

12. Crosby MA, Martin JW, Robb GL, Chang DW. Pediatric mandibular reconstruction using a vascularized fibula flap. Head Neck. 2008; 3 0(3): 311-319. doi: 10.1002/hed.20695. PMID: 17685454.

13. Arnold DJ, Wax MK; Microvascular Committee of the American Academy of Otolaryngology-Head and Neck Surgery. Pediatric microvascular reconstruction: a report from the Microvascular Committee. Otolaryngol Head Neck Surg. 2007; 136 (5): 848-851. doi: 10.1016/j.otohns.2006.11.023. PMID: 17478228.

Correspondencia:

Julio Acero

E-mail: j-acero@telefonica.net 